\title{
LAS ALEXIAS FONOLÓGICA, DE SUPERFICIE Y PROFUNDA EN HISPANO- HABLANTES Y LOS MODELOS DE DOBLE RUTA
}

\section{THE PHONOLOGICAL, SURFACE AND DEEP ALEXIAS IN SPANISH- SPEAKING INDIVIDUALS AND THE DOUBLE ROUTE MODELS}

\author{
Aldo Ferreres \\ Cynthia Valeria López \\ Universidad de Buenos Aires, Argentina
}

\begin{abstract}
Resumen: Se presenta una revisión de casos de alexia en hispanohablantes cuyas características coinciden con los cuadros de alexia fonológica, de superficie y profunda descriptos inicialmente en pacientes que leían en sistemas de escritura "opacos" (como el francés y el inglés). Estos sistemas poseen muchas palabras de ortografía irregular que no pueden ser leídas en voz alta por procedimientos de conversión grafema-fonema y que justifican, entre otras razones, la formulación de los modelos de doble ruta de lectura. Con el argumento de la transparencia del sistema de escritura del español, en el que todas las palabras se pueden leer por procedimientos de conversión grafema-fonema, se ha cuestionado la existencia de los cuadros de alexia fonológica, de superficie y profunda en hispanohablantes y la pertinencia de los modelos de doble ruta para explicarlos (Ardila 1991, 1998). Nuestro estudio aporta evidencia empírica a favor de la pertinencia de la tipología mencionada y del poder explicativo de los modelos de doble ruta de lectura también para pacientes hispanohablantes.
\end{abstract}

Palabras clave: alexia, dislexia adquirida, alexia fonológica, alexia de superficie, alexia profunda, hispanohablantes, paralexias semánticas.

\begin{abstract}
A review of alexia's cases in Spanish-speaking individuals whose characteristics fit with the deep, surface and phonological alexia syndromes, initially described with patients that were reading in "opaque" systems of writing (as French and English) is presented. These systems possess many words of irregular spelling that cannot be read loudly by procedures of conversion grapheme-phoneme and that justify, among other reasons, the formulation of the double route models of reading. Due to the argument regarding the transparency of the Spanish writing system, in which all the words can be read by the procedures of conversion grapheme-phoneme, not only the existence of the reading syndromes mentioned, in Spanish speakers has been questioned, but also the relevancy of the double route models that explain them (Ardila 1991, 1998).

The present study provides with empirical evidence to support the applicability of the mentioned typology, and the explanatory power of the double route models of reading for Spanish-speaking patients.
\end{abstract}

Keywords: alexia, acquired dyslexia, phonological alexia, surface alexia, deep alexia, Spanish-speaking individuals, semantic paralexias.

La publicación de este artículo se ha realizado en el marco del proyecto UBACYT P008.

\section{INTRODUCCIÓN}

A partir de los trabajos de Marshall y Newcombe (1973) se ha desarrollado una intensa investigación en el terreno de las alexias (alteraciones de la lectura adquiridas por lesión cerebral). La introducción de modelos, pruebas y datos provenientes de la investigación psico- lingüística ha permitido reinterpretar los síntomas de la alexia e identificar nuevas tipologías. Los complejos sintomáticos más consensuados fueron designados con los nombres de alexia fonológica, alexia de superficie y alexia profunda. A partir de estos estudios una parte importante de los autores ha defendido los llamados modelos de lectura de doble ruta.

Correspondencia: Aldo Ferreres. Facultad de Psicología, Universidad de Buenos Aires. Correo electrónico: aferreres@psi.uba.ar. 
Los modelos duales (Coltheart, 1985; Coltheart, Curtis, Atkins y Haller, 1993; Ellis, 1982; Patterson y Morton, 1985;) sostienen que la lectura se realiza a través de dos procedimientos: uno léxico y otro no-léxico (Figura 1).

Ambos requieren el reconocimiento de letras pero a partir de ese punto las rutas divergen y utilizan diferentes componentes.

En la ruta léxica las letras pueden activar la representación léxico ortográfica de una palabra conocida para a su vez activar su significado en el sistema semántico. Esta ruta permite un acceso al significado desde la ortografía. La activación puede trasladarse al léxico fonológico de salida y activar la forma fonológica de la palabra correspondiente y los sucesivos procesos que permiten su pronunciación durante la lectura en voz alta.

La ruta léxica sólo permite comprender y leer en voz alta palabras conocidas, es decir, secuencias de letras ya almacenadas en el léxico ortográfico; sin importar que sean palabras de ortografía regular o irregular, la identificación de la secuencia de letras permite activar su significado y su pronunciación.

Algunos autores también han propuesto una ruta léxica directa que une el léxico ortográfico con el léxico fonológico; esta ruta permite la lectura en voz alta de palabras conocidas, sin necesidad de acceder al significado de las mismas (Schwartz, Saffran y Marin, 1980; Blazely, Coltheart y Casey, 2005; para una argumentación en contra: Hillis y Caramazza, 1991).

La ruta no-léxica procede como un sistema de ensamblaje, primero se lleva a cabo la segmentación de las secuencias de letras en unidades adecuadas para aplicar reglas de conversión grafema-fonema. Así, una nopalabra pronunciable como QUETU (no existe en el diccionario del Español esta secuencia de letras) es segmentada en grupos apropiados QU-E-T-U sobre los que se aplican las reglas de conversión que permiten obtener la secuencia de fonemas /k, e, t, u/, a continuación los fonemas son mezclados en sílabas y pronunciados como un todo con acento incluido /'ketu/. Estos últimos procesos se llevan a cabo en el componente designado como nivel fonémico en el modelo de la Figura 1. Hay discusión sobre el tamaño de las unidades subléxicas que procesa esta vía, grafemas, grupos de grafemas o sílabas. Naturalmente, además de no-palabras, la ruta no-léxica permite leer palabras regulares, cuya pronunciación puede derivarse mediante la aplicación de reglas de conversión.
De esta manera, la ruta no-léxica provee un mecanismo directo para la lectura en voz alta de no-palabras y palabras regulares, pero indirecto, fonológicamente mediado, para el acceso al significado de las palabras regulares. La activación semántica se produciría a posteriori del ensamblado fonológico de manera similar a cuando se comprende una palabra oída.

Dentro de los sistemas de escritura alfabéticos se distinguen sistemas "transparentes" y "opacos". Los sistemas "opacos", como el inglés y el francés, poseen palabras irregulares cuya pronunciación no puede derivarse por reglas de conversión grafema-fonema. En el francés por ejemplo, las palabras CHOCOLAT y CHOLERA se pronuncian /čokola/ y /kolera/; la misma secuencia ortográfica $\mathrm{CHO}$ se pronuncia en un caso /čo/ y en otro /ko/ de manera que no pueden ser pronunciadas en forma correcta aplicando reglas, sólo el conocimiento de la palabra completa permite acceder al significado y la pronunciación correcta. En los sistemas opacos la ruta léxica permite leer todas las palabras (regulares e irregulares) mientras que la ruta no-léxica permite leer no-palabras y palabras regulares. En los sistemas de escritura "transparentes" como el español y el italiano no hay palabras irregulares para la lectura (aunque sí para la escritura) y la pronunciación puede derivarse por aplicación de reglas de conversión. Los cuadros de alexia fonológica, de superficie y profunda han sido esgrimidos como evidencia empírica a favor de los modelos de doble ruta. Consideremos en particular la doble disociación alexia fonológica/alexia de superficie.

En la alexia fonológica el signo más prominente es la disociación entre la severa dificultad para leer no-palabras y la capacidad relativa o completamente conservada para leer palabras (Beauvois y Derouesné, 1985; Sartori, Barry y Job, 1984; Berndt, Haendiges, Mitchum y Wayland, 1996; Coltheart, 1996). Los pacientes no pueden leer en voz alta no-palabras como TORI, SANETO pero sí pueden leer palabras como TORO, SANIDAD, CARAMELO. El cuadro ha sido interpretado como una alteración de la ruta no-léxica con preservación relativa de la ruta léxica. En la alexia de superficie se observa el patrón inverso. El cuadro fue inicialmente identificado en sistemas de escritura opacos como el inglés y el francés que poseen numerosas palabras de ortografía "irregular".

Los pacientes mostraban buena lectura de no-palabras y palabras regulares pero severas dificultades con las palabras irregulares con 
las que cometían errores de regularización (Patterson, Marshall y Coltheart, 1985). La alexia de superficie ha sido interpretada como una alteración de la ruta léxica de lectura con preservación de la ruta no-léxica.

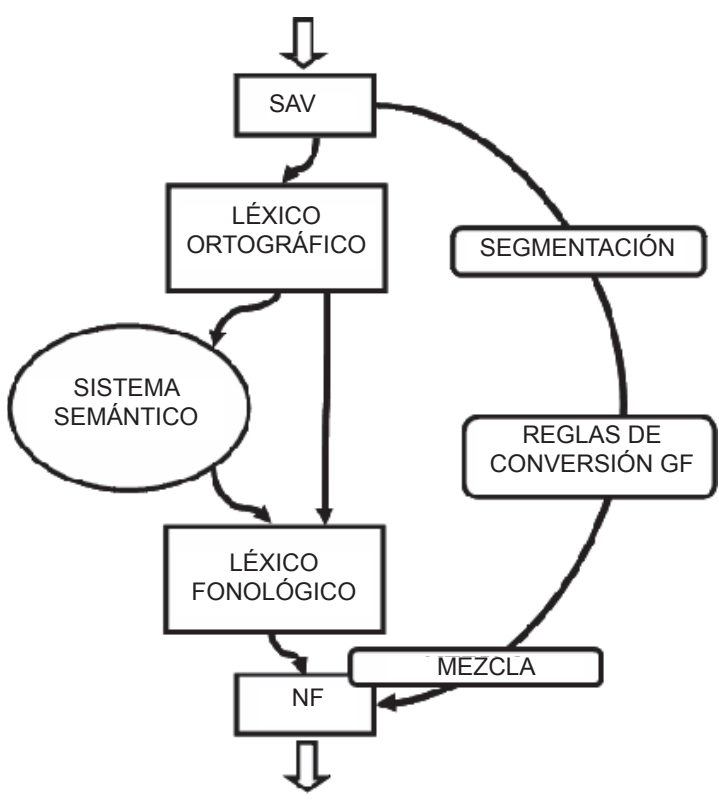

Figura 1. Modelo de doble ruta de lectura

Debido a que la mayor parte de la investigación se realizó en pacientes anglo y franco hablantes y dado que existen diferencias no despreciables entre los sistemas de escritura, se ha planteado la pregunta sobre el valor universal de los hallazgos y la aplicabilidad de los modelos duales a otros sistemas de escritura.

Para el español, se ha puesto en duda la existencia y/o la incidencia de ciertos síntomas como las paralexias semánticas, la conveniencia de recurrir a los tipos de alexia mencionados (fonológica, profunda, de superficie) para describir las alteraciones en hispanohablantes y la aplicabilidad del modelo de doble ruta de lectura. En consonancia con los modelos de "mediación fonológica obligatoria" (Gough, 1972; Van Orden, Johnston y Hale, 1988) se ha cuestionado específicamente la necesidad/ existencia de una ruta léxica en el lector monolingüe español.

Ardila $(1991 ; 1998)$ y Ardila, Rosselli y Pinzón (1989) sostienen que la arquitectura cognitiva funcional de la lectura depende de las características propias de cada sistema de escritura y que la lectura en español está siempre mediada por la fonología y el lenguaje. Además afirman que la existencia de una doble ruta de lectura (fonológica y semántica) tal como fue propuesta para el inglés podría no ser aceptable en español.

Los autores sugieren también que leer en inglés y leer en español representan actividades cognitivas completamente diferentes y por lo tanto, la representación cerebral del lenguaje escrito y los modelos para alexias y agrafias serían, de alguna manera, diferentes. Se sostiene entonces, que la lectura en español se realiza mediante una estrategia grafofonémica y que sólo pueden introducirse estrategias adicionales bajo circunstancias especiales, como el bilingüismo o entrenamientos excepcionalmente altos en lectura (Ardila, 2000).

Efectivamente, el sistema de escritura del español difiere del inglés y el francés debido a su mayor transparencia. Todas las palabras del español son regulares para la lectura y su pronunciación puede ser obtenida mediante procedimientos de conversión grafema-fonema. Pero que todas las palabras del español puedan ser leídas por mecanismos no-léxicos no implica necesariamente que deban ser leídas de esa manera y que esta particularidad del sistema impida el desarrollo de un procedimiento léxico de lectura (más rápido y eficiente).

Las palabras regulares del español, al igual que las palabras regulares e irregulares del inglés y del francés, son secuencias únicas de letras que suenan de cierta manera y tiene un significado particular. En teoría, nada impide que un lector, inglés o español, que se enfrente frecuentemente con estas palabras asocie el agrupamiento de letras con el significado y con la forma fonológica correspondientes desarrollando de esta manera una rutina léxica de lectura (Valle-Arroyo, 1996).

Las particularidades del español introducen también algunos problemas empíricos. Uno de ellos es que la inexistencia de palabras irregulares impide someter a prueba uno de los signos capitales de la alexia de superficie: la producción de errores de regularización en la lectura de palabras irregulares. Sin embargo, Ferreres, Martinez Cuitiño y Olmedo (2005) publicaron recientemente el primer caso de un trastorno adquirido de la lectura en hispanohablantes que sigue el patrón de la alexia de superficie y señalaron las pruebas que permiten poner en evidencia la alteración de la ruta léxica y la preservación de la ruta no-léxica de lectura. 
Los objetivos de este trabajo son:

1. revisar evidencia empírica sobre la existencia de pacientes hispanohablantes con alteraciones de la lectura cuyas características coinciden con los cuadros aléxicos descriptos en pacientes que leían en sistemas de escritura opacos (inglés y francés) y

2. discutir la utilidad de los modelos de doble ruta para interpretar las alexias en español.

Para mayor claridad de la exposición, se consideró conveniente desplazar una parte de la introducción, la correspondiente a consideraciones más detalladas sobre los distintos tipos de alexia, a la sección de resultados, donde también se agrega una discusión parcial sobre cada caso, previa a la discusión general.

\section{MÉTODO}

\section{Casos}

Revisamos tres pacientes hispanohablantes LT, MM y JMK, seleccionados porque las características de la alexia de cada uno de ellos coincide con uno de los cuadros conocidos como alexia fonológica, de superficie y profunda.

El paciente LT, hombre, diestro, ingeniero universitario, dueño y gerente de una farmacia. A la edad de 47 años sufrió un ACV isquémico con leve compromiso motor y sensitivo y afasia. La tomografía computada cerebral mostró una lesión opercular izquierda con extensión a los lóbulos temporal y frontal. Recibió terapia del lenguaje desde el comienzo. El presente estudio se realizó 10 años después del ACV, en ese momento su evaluación del lenguaje con la BADA (Ferreres et al., 1999) mostró buena comprensión auditiva de palabras y oraciones, dificultades anómicas y agramáticas en la producción oral, alexia fonológica y alteraciones leves en memoria de corto plazo fonológica.

El paciente MM, hombre, diestro, abogado universitario y periodista, manejaba inglés y portugués como segunda lengua, sufrió un traumatismo encéfalo craneano (TEC) a la edad de 53 años. A los 4 meses presentaba paresia facial central derecha leve, hemianopsia derecha, y un cuadro de afasia de Wernicke con importantes dificultades de comprensión auditiva y visual, anomia, jergafasia con pa- rafasias y neologismos, sin déficits fonéticos. La tomografía de cerebro mostró una lesión témporo-parietal izquierda.

El estudio de su alexia se realizó tres años después momento en el que su lenguaje analizado con la BADA mostró un cuadro de anomia severa, agramatismo expresivo sin déficits fonéticos, leves dificultades sintácticas y semánticas en la comprensión auditiva y déficit de memoria de corto plazo verbal. Mostraba lentitud en la lectura y errores ortográficos en escritura.

El paciente JMK, hombre, diestro, bilingüe español-esloveno (nació en Buenos Aires, sus padres eran inmigrantes provenientes de $\mathrm{Yu}$ goslavia), hizo la escuela primaria en español y la escuela secundaria, parte en esloveno (en Yugoslavia) y parte en español. Completó 12 años de escolaridad. A la edad de 50 años sufrió un accidente cerebro vascular hemorrágico con hemiplejía y afasia y fue operado para tratar un aneurisma en la arteria silviana izquierda. La tomografía computada cerebral posquirúrgica mostró un extenso infarto fronto-parieto- temporal izquierdo. Fue evaluado en nuestro servicio cinco años después de iniciado el cuadro.

El examen neurológico mostró una hemiparesia y hemihipoestesia derechas leves, sin hemianopsia, y apraxia de miembros que afectaba sobre todo la mano derecha.

El examen del lenguaje mostró una severa alteración de la expresión verbal debido a anomia y agramatismo; producía palabras yuxtapuestas o frases simples con omisión de palabras funcionales y poca utilización de verbos, generalmente en infinitivo; conservaba una buena articulación del habla y sólo ocasionalmente producía errores fonémicos o fonéticos, el habla era disprosódica tanto en repetición como en producción espontánea. La denominación de dibujos estaba severamente afectada pero podía repetir bien sílabas, palabras y oraciones simples y producía errores de tipo agramático en la repetición de oraciones complejas. La comprensión auditiva estaba mejor pero no indemne, la comprensión de palabras aisladas era bastante buena pero la comprensión de la estructura sintáctica de las oraciones estaba muy afectada. La lectura y la escritura estaban severamente afectadas. Realizaba bien el emparejamiento de alógrafos pero no el emparejamiento auditivo-visual fonema-grafema; el emparejamiento palabra escrita/dibujo estaba afectado ( $40 \%$ de acierto) mientras que el emparejamiento palabra oída/ 
dibujo con los mismos estímulos estaba conservado. La sonorización de grafemas, sílabas y palabras escritas era imposible. Salvo su nombre, la escritura estaba reducida a la copia servil. El dictado y la escritura espontánea estaban completamente abolidos. Si bien los casos de LT y MM fueron analizados en publicaciones recientes (Ferreres y cols, 2005; Ferreres, 2008), el tercer paciente, JMK, fue estudiado y publicado con anterioridad (Ferreres y Miravalles, 1995). Por tal motivo, incluimos algunos datos de este caso porque son relevantes para la presente discusión y porque desde esa fecha no hemos tenido oportunidad de estudiar ningún otro paciente con un cuadro aléxico como el suyo. Por esta razón, las pruebas con las que se estudió a JMK difieren de las que utilizamos con LT y MM. El estudio de pacientes LT y MM consistió en: a) una exploración general del lenguaje para caracterizar el cuadro afásico que se efectuó con la BADA (Batería para el Análisis de los Déficits Afásicos, Ferreres et al., 1999) y b) pruebas experimentales para caracterizar las alteraciones de lectura.

Lectura de palabras y no-palabras: se utilizó la prueba diseñada por China y Ferreres (1998) que consta de 45 palabras y 45 no-palabras. Las 45 palabras son sustantivos concretos dibujables controlados en cuanto a longitud y frecuencia. La frecuencia de las palabras fue obtenida del Diccionario de Frecuencias de las Unidades Lingüísticas del Castellano de Alameda y Cuetos (1995). Se controló la complejidad silábica (30 palabras que incluyen una sílaba no-CV y 30 que incluyen sólo sílabas $C V$ ).

La lista de no palabras se construyó mediante la recombinación de las sílabas de las palabras, tratando de que resultaran no-palabras lejanas de palabras reales. Las no-palabras fueron emparejadas con las palabras en cuanto a la longitud y complejidad silábica. Las palabras y no-palabras fueron leídas en listas separadas.

Cada estímulo se presentó en una tarjeta independiente con letra de imprenta minúscula de tamaño grande (36 ps.), no se utilizó restricción de tiempo y se contabilizó la mejor respuesta del paciente o aquella que él consideraba su respuesta final.

Los errores en la lectura de palabras fueron clasificados en:

a) palabras relacionadas, subclasificados en: errores semánticos, visuales, morfológicos, otros (corresponden a las paralexias semánticas, visuales y morfológicas res- pectivamente);

b) palabras no relacionadas;

c) no-palabras relacionadas y

d) no-palabras no relacionadas.

Los errores en la lectura de no-palabras

fueron clasificados en:

a) palabras relacionadas,

b) no-palabras relacionadas,

c) no-palabras no relacionadas.

Prueba de decisión léxica con no-palabras corrientes y pseudohomófonos: para evaluar si el reconocimiento de las palabras se realizaba a partir de información ortográfica o fonológica se diseñó una prueba de decisión léxica con pseudohomófonos. La prueba incluyó 60 palabras, 30 no-palabras "corrientes" y 30 pseudohomófonos. La no-palabras "corrientes" fueron obtenidas por sustitución de una letra de una palabra, ejemplo: cunebra (proveniente de culebra), alivo (olivo), mertuza (merluza) de manera que se distinguían de una palabra tanto ortográfica como fonológicamente.

Por otro lado, los pseudohomófonos son nopalabras homófonas de palabras. En español, como en otras lenguas, es posible seleccionar secuencias de letras que no corresponden a palabras ortográficamente legales pero cuya pronunciación coincide con palabras fonológicas de la lengua (ejemplo: votella, camiza, relijión, fucil). Estas no-palabras resultan homófonas de palabras fonológicas (botella, camisa, religión, fusil) porque se obtuvieron mediante el cambio de una letra por otra que según las reglas de conversión de la lengua se pronuncian igual ( $b>v, s>z, g>j, s>c$, en los ejemplos anteriores). Tanto las no-palabras "corrientes" como los pseudohomófonos se distinguen de las palabras ortográficas por la sustitución de una letra. Pero, mientras la secuencia fonológica de las no-palabras corrientes difiere de la fonología de las palabras de las que fueron derivadas (mertuza-merluza), la secuencia fonológica de un pseudohomófono es idéntica a la secuencia fonológica de la palabra de la que fue derivado (fucil-fusil). Si la decisión léxica de un paciente se apoya en el procesamiento ortográfico del estímulo, es de esperar que el rendimiento en no-palabras corrientes y pseudohomófonos sea similar. En cambio, si la decisión léxica se apoya en el procesamiento fonológico, es decir, se realiza luego de una mediación fonológica, el paciente no tiene forma de distinguir entre una palabra real y un pseudohomófono, lo que conducirá a falsos positivos con estos últimos. 


\section{Pruebas que evalúan la vía no-léxica de lectura y el procesamiento fonológico}

a) Prueba de segmentación grafémica: Se evaluó la segmentación ortográfica mediante una tarea de contar fonemas. Se presentaron 18 tarjetas con una no-palabra de tres letras escrita, monosilábica, la mitad de los estímulos correspondían a dos fonemas y la otra mitad a tres fonemas (ejemplo: GUE /ge/ y PRA/pra/), el paciente debía decir si el estímulo escrito tenía dos o tres fonemas.

b) Prueba reglas de conversión ortografíafonología: el dominio de las reglas de conversión ortografía-fonología fue evaluado a nivel de grafemas aislados y de sílabas.

i) En la tarea de conversión grafemafonema se presentaron 28 grafemas aislados escritos en tarjetas y los pacientes tenían que dar su sonido (Ejemplo: $\mathrm{S}=/ \mathrm{s} /$; $\mathrm{LL}=/ \mathrm{y} / ; \mathrm{B}=/ \mathrm{b} / ; \mathrm{CH}=/ \mathrm{c} /$ ).

ii) Como teóricamente la conversión ortografía-sonido también podría operar con unidades silábicas (mediante asociaciones sílaba escrita - sílaba pronunciada) también se tomó la pronunciación de 36 sí labas CV (consonante vocal) cada una de ellas escrita en una tarjeta separada.

c) Pruebas de mezcla (blending) de fonemas y mezcla de sílabas:

i) En la tarea de mezclar fonemas el eva luador pronuncia dos o tres fonemas, uno a uno, y el paciente debe unirlos para pronunciarlos juntos, como una única sí laba (ejemplo: /g/ /a//n/ > /gan/); se presen taron 60 estímulos. Es importante señalar que al escuchar los fonemas separadamente el paciente no recibe información sobre la estructura silábica que debe utilizar para la emisión. Se supone que esta tarea evalúa el proceso de "blending" o ensamble de los fonemas obtenidos a partir de la aplicación de las reglas de con-versión. Sin duda es una ta rea metalingüística y poco natural pero los con troles con el nivel de escolaridad de nuestros pa cientes lograron un $94,7 \%$ de respuestas correctas.

ii) En la tarea de mezclar sílabas el pa ciente oye 2 o 3 sílabas que el evaluador pronuncia separadas, una a una, y debe pronunciarlas juntas, en una única emi sión. Es una tarea más natural que la ante- rior y se encuentra facilitada porque el estímulo auditivo ya proporciona la estructura silábica.

d) Pruebas de memoria de corto plazo fonológica: evaluada mediante las tareas de repetición de series de palabras y no palabras de la BADA:

i) para determinar el span de palabras se presentan 10 series de 2 y 3 palabras y 5 se ries de 4 y 6 palabras, el paciente debe escu char cada serie y luego repetirla en el mismo orden; para que una determinada longitud de serie se compute como correcta el pa ciente debe repetir bien el $50 \%$ o más de las series de esa longitud determinada, el span corresponde a la mayor longitud en la que el repite correctamente el $50 \%$ o más de las series (por ejemplo si repite bien el $60 \%$ de las series de 3 palabras y el $40 \%$ de las series de 4 palabras tiene un span de 3 ); ii) para determinar el span de no-palabras se procede de manera similar pero se utilizan 10 series de 2 y 3 no-palabras y 1 serie de 4 no-palabras.

\section{Pruebas que evalúan el procesamiento semántico}

Prueba: para estimar el procesamiento semántico se analizó el rendimiento de los pacientes en las tareas de emparejamiento palabra oída-dibujo (40 sustantivos, 20 verbos) y palabra escrita-dibujo (40 sustantivos, 20 verbos) que son pruebas de la BADA (Ferreres y col., 1999). En esta tarea el paciente escucha o lee una palabra y tiene que elegir, entre dos dibujos, el que corresponde a la palabra oída o leída; en la mitad de los estímulos el segundo dibujo corresponde a un distractor semántico (ejemplos: oreja - ojo; esquiar - patinar) y en la otra mitad a un distractor relacionado por la forma de la palabra (fonológica o visual, ejemplos: bombilla - sombrilla; rezar - remar).

\section{Pruebas que evalúan la escritura al dictado}

a) Prueba de dictado de palabras y nopalabras: se utilizaron los mismos estímulos que en la prueba de lectura de palabras y no-palabras pero esta vez en una tarea de dictado.

b) Prueba de dictado de palabras regulares e irregulares: se diseñó una prueba de 
dictado que incluye123 palabras de ortografía regular para la escritura, palabras cuya escritura puede derivarse sin ambigüedades aplicando reglas de conversión fonema - grafema (ejemplos: COPA, MODERNA, PUERTO, MORADO) y 117 palabras de ortografía irregular (cuya escritura correcta no puede derivarse por reglas y requiere el conocimiento de la palabra, ejemplos: NIEVE, VERSIÓN, CABALLO, CAMISA; la escritura de estos estímulos mediante reglas de conversión puede originar errores fonológicamente plausibles como NIEBE, BERSIÓN, CABAYO, CAMIZA). Sólo se aplicó la prueba a MM. JMK fue evaluado con una lista de 250 palabras aisladas de distinta categoría gramatical, 50 nombres concretos y 50 abstractos, 50 verbos conjugados, 50 adjetivos, 25 adverbios y 25 palabras funcionales (Dubrovsky y cols. 1990). Dado que carecíamos de un diccionario de frecuencias en el momento de diseñar la prueba, esta variable no estaba controlada. La lectura de no-palabras fue explorada con una lista de 60 estímulos. Se analizó el porcentaje de respuestas correctas, de sustituciones y de omisiones en la lectura de palabras y no-palabras. En el caso de la lectura de palabras, las sustituciones pueden ser paralexias (error en el que la palabra escrita es leída en voz alta como otra palabra). Para la clasificación de paralexias utilizamos la propuesta de Coltheart (1980), algo modificada. Esta lista incluye:

i) paralexias semánticas (la palabra pronunciada por el paciente está semánticamente relacionada con el estímulo escrito), ii) paralexias morfológicas (sustituciones por palabras morfológicamente relacionadas), iii) paralexias visuales (visual u ortográfica mente relacionadas -la palabra sustituyente posee más del $50 \%$ de las letras del estímulo-), iiii) paralexias visuales ocultas (en las que se puede inferir que se superpo nen un error visual y luego uno semántico), iiiii) sustitución por funcionales (una palabra funcional es sustituida por otra de la misma clase gramatical).

La comprensión visual de palabra aislada se exploró con una prueba de 10 estímulos escritos, en la que se le presentaba al paciente una tarjeta con una palabra escrita y el paciente debía señalar el dibujo correspondiente en una lámina con 20 dibujos (10 blancos y 10 distractores semánticos y visuales). Para el análisis se tomaron también en cuenta los rendimientos en las tareas de lectura que formaban parte de la exploración clínica de su afasia.

\section{RESULTADOS}

Se reportan los resultados de cada paciente precedidos por consideraciones sobre el tipo respectivo de alexia.

\section{Alexia fonológica}

El signo más prominente de la alexia fonológica es la disociación entre una severa dificultad para leer no-palabras y una capacidad relativa o completamente conservada para leer palabras.

Los pacientes no pueden leer en voz alta no-palabras como TORI, SANETO, etc., mientras sí pueden leer palabras como TORO, SANIDAD, CARAMELO, etc. Beauvois y Derouesné (1979) describieron el primer caso de alexia fonológica: el paciente leía correctamente el $94 \%$ de las palabras y sólo el $10 \%$ de las nopalabras. Otros pacientes comunicados posteriormente mostraron un rendimiento variable en la lectura de palabras y no-palabras. Sartori, Barry y Job (1984) revisaron los 16 pacientes publicados hasta la fecha de su comunicación y encontraron que los porcentajes de lectura correcta de palabras oscilaban entre 38 y 100 $\%$ y los de no-palabras entre el 0 y el $80 \%$. Los errores en la lectura de palabras fueron paralexias visuales y morfológicas y muy pocas o ninguna paralexia semántica. Los errores en la lectura de no palabras fueron principalmente lexicalizaciones (sustituye la no-palabra por una palabra relacionada: murata $>$ mulata) y omisiones.

Algunos pacientes, pero no todos, mostraron efectos de frecuencia, clase y/o categoría gramatical en la lectura de palabras. Estos datos sugieren que la alexia fonológica no es tampoco un síndrome homogéneo, sin embargo se sigue utilizando el término para referirse a pacientes que muestran mejor rendimiento en la lectura de palabras que en no-palabras, reconociendo la variabilidad existente tanto en la lectura de palabras como en la lectura de no-palabras (Berndt, Haendiges, Mitchum y Wayland, 1996).

La denominación alexia fonológica no se usa con la pretensión de distinguir un síndrome homogéneo, sino como una manera cómoda de designar a un conjunto heterogéneo de pacientes cuyo rasgo común es presentar una alteración de la lectura no-léxica (Coltheart, 1996). 
Existen varios casos de alexia fonológica en hispanohablantes publicados hasta la fecha (Cuetos, Valle-Arroyo y Suárez, 1996; Dansilio \& Dalmás, 1997; Ferreres, López, Petracci y China, 2003; Ferreres, Martinez Cuitiño, Jacubovich, Olmedo y López, 2003; Ferreres, 2008). Sin embargo este bajo número parece reflejar más los problemas metodológicos en el estudio de los pacientes en nuestro medio, que la incidencia real de la alteración.

\section{El caso $L T$}

Lectura en voz alta de palabras y nopalabras: LT leyó correctamente todas las palabras pero mostró severas dificultades para leer no-palabras y la diferencia en eficacia fue significativa. Sus tiempos de lectura estaban prolongados, tanto para palabras como para nopalabras pero LT mantuvo $\mathrm{y}$, más precisamente, "exageró" la ventaja léxica ya que su media de tiempo en la lectura de no-palabras resultó casi 23 veces mayor que la de los controles. Aunque la media en el tiempo de lectura de palabras en LT duplicó el que utilizaron los controles, las palabras fueron leídas pronunciándolas como un todo, sin proceder sílaba a sílaba.

LT mostró una clara disociación en la eficacia para leer palabras y no-palabras y en sus tiempos de lectura (tabla 1). La disociación entre la lectura de palabras conservada y la lectura de no-palabras alterada sugiere que ambos tipos de estímulos fueron leídos por mecanismos diferentes y que el mecanismo no-léxico resultó más afectado por la lesión que el mecanismo léxico. Estos datos permiten encuadrar el déficit de lectura de LT dentro del complejo sintomático de la alexia fonológica.

Tabla1. Lectura de palabras y no-palabras

\begin{tabular}{|c|c|c|c|c|c|}
\hline \multicolumn{2}{|c|}{ LT } & \multicolumn{3}{|c|}{ MM } & Controles \\
\hline N & $\%$ & N & \multicolumn{2}{|c|}{$\%$} & $\%$ \\
\hline Palabras correctas/45 & 45 & 100,00 & 44 & 97,80 & 100,00 \\
\hline $\begin{array}{c}\text { No-palabras } \\
\text { correctas/45 }\end{array}$ & 27 & 60,00 & 44 & 97,80 & 100,00 \\
\hline Diferencia P-NP & \multicolumn{2}{|c|}{ si $^{* * *}$} & \multicolumn{2}{|c|}{ n.s. } & n.s. \\
\hline
\end{tabular}

P: palabras. NP: no-palabras.

Decisión léxica visual con no-palabras corrientes y pseudohomófonos: LT no tuvo dificultad con los pseudohomófonos, los rechazó correctamente con un porcentaje de acierto incluso superior al que logró con las no-palabras. La prueba de chi cuadrado mostró una diferencia significativa entre los distintos estímulos pero en LT la causa de la diferencia no fueron los pseudohomófonos sino un menor rendimiento con las palabras. LT rechazó erróneamente 11 palabras (falsos negativos), la mayoría de baja frecuencia y escritura irregular (ejemplos de palabras erróneamente rechazadas: HADA, PABELLÓN, ESBOZO). Esto sugiere que si bien $L T$ conserva información léxico ortográfica como para rechazar la inmensa mayoría de los pseudohomófonos ésta no es suficiente como para reconocer palabras de baja frecuencia.

Emparejamiento palabra escrita/dibujo y palabra oida/dibujo: en esta prueba LT logró el $100 \%$ de respuestas correctas, tanto para objetos como para acciones. Las respuestas fueron rápidas.

Pruebas que evalúan la vía no-léxica de lectura y el procesamiento fonológico (tabla 2): tanto en la prueba de reconocimiento de letras como en la segmentación grafémica LT tuvo buen rendimiento no mostrando dificultades para las mismas. En las pruebas de conversión ortografía-fonología tuvo un rendimiento bajo aunque la conversión de sílabas estuvo mejor que la de grafemas. En las pruebas de ensamblaje de fonemas obtuvo solo un $30 \%$ de aciertos y si bien el ensamblaje de sílabas estuvo mejor, ambos rendimientos fueron inferiores al de los controles.

Tabla 2. Pruebas que evalúan reconocimiento de letras, mecanismos de lectura no-léxica, procesamiento fonológico y semántico

\begin{tabular}{|c|c|c|c|c|c|}
\hline \multicolumn{2}{|l|}{$L T$} & \multicolumn{3}{|c|}{ MM } & \multirow{3}{*}{\begin{tabular}{|c|}
$\begin{array}{c}\text { Contro- } \\
\text { les }\end{array}$ \\
$\%$ \\
98,40
\end{tabular}} \\
\hline$n$ & $\%$ & $n$ & \multicolumn{2}{|c|}{$\%$} & \\
\hline $\begin{array}{l}\text { E } m p \text { a } r \text { e } j . \\
\text { Alógrafos } / 25\end{array}$ & 25 & 100,00 & 25 & 100,00 & \\
\hline $\begin{array}{l}\text { Segmentación } \\
\text { ortográfica/18 }\end{array}$ & 18 & 100,00 & 17 & 94,40 & 98,90 \\
\hline $\begin{array}{l}\text { Conversión G- } \\
\text { F/28 }\end{array}$ & 24 & 85,70 & 15 & 53,60 & 100,00 \\
\hline $\begin{array}{l}\text { Conversión } 0-\mathrm{F} \\
\text { sílabas/36 }\end{array}$ & 34 & 94,40 & 29 & 80,60 & 100,00 \\
\hline $\begin{array}{ll}\text { Mezcla } & \text { de } \\
\text { fonemas/60 }\end{array}$ & 18 & 30,00 & 30 & 50,00 & 94,70 \\
\hline $\begin{array}{ll}\begin{array}{l}\text { Mezcla } \\
\text { sílabas/40 }\end{array} & \\
\end{array}$ & 37 & 92,50 & 32 & 80,00 & 99,50 \\
\hline MCP span P & \multicolumn{4}{|c|}{$4 \mathrm{P}$} & $2 \mathrm{P}$ \\
\hline MCP span NP & \multicolumn{2}{|c|}{$2 \mathrm{NP}$} & & & $1 \mathrm{NP}$ \\
\hline Repetición P/45 & 45 & 100,00 & 45 & 100,00 & 100,00 \\
\hline $\begin{array}{l}\text { Rep e tición } \\
\text { NP/45 }\end{array}$ & 43 & 95,60 & 39 & 86,70 & 100,00 \\
\hline Dif.Repet P-NP ${ }_{a}$ & \multicolumn{2}{|c|}{ n.s. } & \multicolumn{2}{|c|}{ n.s. } & No \\
\hline $\begin{array}{l}\text { Emp. P Oída-Di- } \\
\text { bujo (BADA)/60 }\end{array}$ & 60 & 100,00 & \multicolumn{2}{|c|}{57} & 95,00 \\
\hline $\begin{array}{l}\text { Emp. P Leída-Di- } \\
\text { bujo (BADA)/60 }\end{array}$ & 59 & 98,33 & \multicolumn{2}{|c|}{57} & 95,00 \\
\hline
\end{tabular}

P: palabras. NP: no-palabras 
En cuanto a las pruebas de MCP fonológica LT mostró bajo rendimiento en el span de palabras, alcanzó el $80 \%$ de correctas en las series de 2 palabras y $40 \%$ en las series de tres palabras (span de palabras $=2$ ); en las series de 2 no-palabras alcanzó el $90 \%$ y en las 3 nopalabras el $40 \%$ (span de no-palabras $=2$ ).

No se cuenta con normas fiables pero el span de palabras se calcula en $6 \pm 2$ y se considera que el span de no-palabras es de menor magnitud, entre 3 y 4 . En cambio el rendimiento fue bueno en la prueba de repetición de palabras.

Pruebas que evalúan la escritura al dictado: en la prueba de dictado de palabras y no palabras el rendimiento fue levemente menor en el dictado de no-palabras presentando solo errores de acentuación (tabla 3).

Tabla 3. Pruebas que evalúan la escritura al dictado

\begin{tabular}{|c|c|c|c|c|}
\hline \multicolumn{2}{|c|}{ LT } & \multicolumn{3}{c|}{ MM } \\
\hline$n$ & $\%$ & \multicolumn{2}{|c|}{$n$} & $\%$ \\
\hline Dictado P/45 & 39 & 86,70 & 40 & 88,90 \\
\hline Dictado NP/45 & 35 & 77,80 & 36 & 80,00 \\
\hline Dif. P - NP & \multicolumn{3}{|c|}{ No } & \multicolumn{2}{c|}{ No } \\
\hline P $^{*}$ Regulares/123 & \multicolumn{2}{|c|}{109} & 90,80 \\
\hline P*Irregulares/117 $^{*} 81$ & 67,50 \\
\hline Dif. PR - PI & \multicolumn{3}{|c|}{ Si $^{* * *}$} \\
\hline
\end{tabular}

P: palabras. NP: no-palabras.

PR: palabras regulares. PI: palabras irregulares.

\section{Discusión del caso LT}

LT mostró una clara disociación entre una buena lectura de palabras y una pésima eficacia en la lectura de no-palabras. El paciente leyó las no-palabras a través de una laboriosa estrategia compensatoria, probablemente obtenida a través de su tratamiento rehabilitador.

El procedimiento no automatizado que utilizaba LT demandaba una gran cantidad de recursos cognitivos ya que requería múltiples recuperaciones léxicas y la utilización de habilidades fonológicas para segmentar la primera sílaba de cada una de las palabras evocadas. El alto consumo de recursos de esta estrategia se manifestó en la exagerada prolongación del tiempo de lectura de las no-palabras que fue 23 veces superior al de los normales.

La afirmación de que la lectura en Español se realiza por mediación fonológica obligatoria no puede explicar estos hallazgos ya que, a pesar de las severas dificultades para leer nopalabras (estímulos que sólo pueden leerse por mediación fonológica) podía leer correctamente todas las palabras de la lista en un tiempo notoriamente inferior.

El paciente nunca utilizó la estrategia compensatoria con las palabras, simplemente podía obtener su pronunciación correcta a partir del estímulo escrito. La estrategia compensatoria que utilizó en sus intentos de leer no-palabras es reveladora: no podía extraer la información fonológica de la primera sílaba de la no-palabra pero sí podía recuperar la representación ortográfica de una palabra que comenzara con la misma sílaba.

Para compensar un déficit de mediación fonológica utilizaba... jun mecanismo léxico ortográfico! El modelo de doble ruta permite una explicación más plausible del patrón aléxico de LT asumiendo que la lesión afectó severamente el funcionamiento de la ruta no-léxica respetando relativamente el de la ruta léxica.

¿A qué nivel o niveles estaba afectada la ruta no-léxica en LT? Los resultados sugieren que el reconocimiento y la segmentación de letras no estaban afectados y que el dominio de las reglas de conversión grafema-fonema aunque afectado, mostraba un grado importante de eficacia ( $85,7 \%$ de acierto en la tarea).

En cambio hay dos componentes severamente afectados en LT, la mezcla de fonemas (blending) y la MCP fonológica que es el espacio de memoria en que se realiza la mezcla. Así, aunque LT obtuviera una importante cantidad de fonemas mediante la aplicación de reglas de conversión grafema-fonema, no podía leer no-palabras porque no podía combinar los fonemas en sílabas pronunciables. En esta línea de razonamiento, el buen rendimiento en la tarea de conversión ortográfico fonológica de sílabas debe analizarse con precaución; en efecto, no pudo ser obtenido mediante una conversión grafema-fonema (bastante conservada) seguido de la mezcla debido a la severa afectación de este último proceso. Más probable es que la conversión ortográfico fonológica de sílabas haya sido realizada exitosamente por LT a través de su estrategia de evocar palabras y segmentar su primera sílaba, estrategia facilitada por el hecho de que para esta tarea se utilizaron sílabas $\mathrm{CV}$ aisladas. En la tarea de decisión léxica la eficacia de LT se asemeja a la de los controles y se dife rencia netamente de la de MM. 
En efecto, LT no produjo errores con los pseudohomófonos lo que obliga a concluir que realizó la tarea apoyándose en la información léxica ortográfica y no en la información fonológica.

Un dato que apoya la afirmación anterior es que las latencias para rechazar las no-palabras fueron más breves que las latencias para aceptar palabras y para rechazar pseudohomófonos; esto contrasta con los tiempos de lectura en voz alta donde las no-palabras consumían casi 23 veces más tiempo que las palabras.

LT no recurre a la fonología para realizar la decisión léxica, ni siquiera para rechazar las nopalabras, de lo contrario serían estos estímulos los que mostrarían mayores latencias. Podemos explicar los resultados de LT asumiendo que realiza la tarea de decisión léxica mediante una consulta con su memoria ortográfica de las palabras, es decir mediante el ingreso directo de la información al componente denominado léxico ortográfico.

\section{Alexia de superficie}

La alexia de superficie fue identificada en sistemas de escritura opacos como el inglés y el francés que poseen numerosas palabras de ortografía "irregular". La característica principal es la disociación entre las severas dificultades del paciente para leer palabras irregulares y la conservación de la lectura de palabras regulares y no-palabras (Patterson, Marshall y Coltheart, 1985). Otras características son: la producción de errores de regularización en la lectura de palabras irregulares (leen las palabras irregulares como si fueran regulares); el reconocimiento y la comprensión mediados por la pronunciación y no por la forma ortográfica; las dificultades en la comprensión de homófonos (CASA-CAZA) y también, los errores de omisión, sustitución e intercambio de letras.

En una primera aproximación se podría pensar que la alexia de superficie no existe, o al menos es indetectable, en lenguas "transparentes" como el español o el italiano, en las que todas las palabras son regulares para la lectura y por lo tanto, la pronunciación siempre puede derivarse por aplicación de reglas. El único caso de alexia de superficie en hispanohablantes publicado es FE (Masterson, Coltheart y Meara, 1985) pero su estudio ofrece dudas debido a su bilingüismo y a las pruebas utilizadas. Se han publicado casos de pacientes italianos pero con una metodología de difícil aplicación al español. Un caso de alexia de superficie en español comunicado por Ruiz, Cid, Mantiñan, Recalde y Tetelboin (1994) fue revisado por Valle-Arroyo (1996) quien analizó las dificultades metodológicas para poner en evidencia la alexia de superficie en español.

\section{El caso MM}

Lectura en voz alta de palabras y nopalabras: MM no mostró diferencias significativas en la eficacia para leer palabras y no-palabras, rindió casi al tope con ambos estímulos. Los tiempos de lectura estaban prolongados pero para ambos tipos de estímulos, sin diferencia significativa entre las medias de tiempo. La ausencia de diferencias en la eficacia y la desaparición de la ventaja léxica en el tiempo de lectura sugieren que MM leyó ambos tipos de estímulos a través de un único procedimiento no-léxico. Estos datos sugieren que su déficit de lectura puede corresponder a una alexia de superficie (tabla 1). El análisis cualitativo de la lectura de MM mostró que leía tanto palabras como no-palabras de manera escandida, sílaba a sílaba. Pero había una diferencia, mientras con las no-palabras la respuesta final era escandida (ejemplo: RELÍCADE > re-li-ca-de), con las palabras la pronunciación escandida fue seguida en todos menos uno de los casos por la pronunciación normal de la palabras, incluyendo su acento fonológico (BOLSILLO > bol-si-llo ... bolsillo), sólo presentó un error de acento (PELÍCANO > pe-li-ca-no ... pelicano).

Decisión léxica visual con no-palabras corrientes y pseudohomófonos: el paciente tuvo el mismo rendimiento, bastante adecuado, en palabras y no-palabras corrientes $(87 \%$ de respuestas correctas en ambas). Pero con pseudohomófonos el rendimiento fue muy malo, prácticamente a nivel del azar $(47 \%$ de correctas) (tabla 4).

Tabla 4. Decisión léxica con palabras, no-palabras y pseudohomófonos

\begin{tabular}{|c|c|c|c|c|c|}
\hline \multicolumn{2}{|c|}{ LT } & \multicolumn{2}{c|}{ MM } & Controles \\
\hline$n$ & \multicolumn{2}{|c|}{$\%$} & $n$ & $\%$ & $\%$ \\
\hline P correct./60 & 49 & 81,30 & 56 & 93,30 & 97,00 \\
\hline NP correct./30 & 29 & 96,70 & 27 & 90,00 & 97,30 \\
\hline PSH correct./30 & 29 & 96,70 & 10 & 33,30 & 98,00 \\
\hline Dif en eficacia & a & \multicolumn{2}{|c|}{ si $^{*}$ peor en P } & \multicolumn{4}{|c|}{ si $^{* * *}$ peor en PSH } & n.s. \\
\hline Patrón de Error & F- con P baja frec. & \multicolumn{3}{c|}{ F+ con PSH } \\
\hline
\end{tabular}

P: palabras. NP: no-palabras 
La mayoría de los errores en esta prueba fueron los falsos positivos con pseudohomófonos, es decir, el paciente consideró como palabras reales a casi la mitad de las no-palabras homófonas de palabras reales (pseudohomófonos).

Emparejamiento palabra escrita/dibujo y palabra oída/dibujo: MM produjo 6 errores/120 estímulos, tres de los cuales fueron errores semánticos (cepillo>peine; oreja>ojo; tenedor>cuchara).

Si bien el número de errores semánticos es pequeño, es necesario señalar que la tarea de emparejamiento palabras aislada - dibujo sólo suele verse alterada en pacientes cuya afasia sea moderada o severa. Además en MM el cuadro inicial fueuna afasia de Wernicke con déficit semántico (tabla 2).

Pruebas que evalúan la vía no-léxica de lectura y el procesamiento fonológico (tabla 2): en el reconocimiento de letras y segmentación de grafemas el rendimiento fue del $100 \%$. En cambio en las pruebas de conversión grafemafonología tuvo un rendimiento bajo rindiendo peor que los sujetos control.

En las pruebas de mezcla de fonemas el rendimiento fue del $50 \%$ y si bien el rendimiento a nivel de la sílaba fue mejor sigue estando por debajo del rendimiento de los controles. MM también mostró notoria reducción tanto del span de palabras como del span de no-palabras; alcanzó el $70 \%$ de correctas en las series de 2 palabras pero no llegó al $50 \%$ de correctas en las series de tres palabras; en las series de 2 no-palabras apenas logró el $30 \%$ y se 472 le asignó 1 porque su repetición de no-palabras aisladas alcanzó el 86\% (ver más abajo).

Los resultados sugieren existe un compromiso de la capacidad de su memoria de memoria de corto plazo fonológica. En la prueba de repetición de palabras y no-palabras tuvo buen rendimiento si bien cometió errores en la repetición de no-palabras aunque sin mostrar una diferencia significativa con la repetición de palabra (Tabla 3), los errores recayeron en las no-palabras largas, que son las que más exigen memoria de trabajo fonológica ya que la nopalabra oída se debe mantener en la memoria de corto plazo hasta su repetición.

Pruebas que evalúan la escritura al dictado: prueba de dictado de palabras y no palabras el rendimiento fue levemente menor en el dictado de no-palabras presentando solo errores de acentuación (tabla 3).
El análisis cualitativo de los errores mostró que $\mathrm{MM}$, en el dictado de palabras, cometió errores fonológicamente plausibles(ejemplos: BOLSILLO>BOLSIYO, B E R E N J E N A > B E R E N G E N A, MARIPOSA>MARIPOZA).

En la prueba de dictado de palabras regulares e irregulares MM mostró peor rendimiento, estadísticamente significativo, con las palabras irregulares en las que cometió errores fonológicamente plausibles lo que se corresponde con un patrón de agrafia de superficie.

\section{Discusión del caso MM}

MM mostró un rendimiento casi idéntico en la lectura en voz alta de palabras y nopalabras: alcanzó un buen rendimiento para ambos tipos de estímulos, pero con ambos tipos de estímulos la lectura fue lenta, silabeada y con ensayos subvocálicos. En cambio, MM falló en aquellas tareas que requieren el conocimiento ortográfico de las palabras. Los procedimientos de lectura utilizados por MM, aunque lentos y laboriosos, le resultaron suficientes para obtener la pronunciación correcta de las palabras del español (todas regulares) y de las no-palabras, pero lo condujeron a errores en la decisión léxica con pseudohomófonos. Pese a que la regularidad del sistema de escritura hace imposible explorar el síntoma más característico de la alexia de superficie (la disociación entre la lectura de palabras regulares e irregulares), la prueba de decisión léxica con pseudohomófonos permite superar esa dificultad. En efecto, sólo el conocimiento de la ortografía correcta de la palabra le hubiera permitido al paciente evitar los errores que induce la mediación fonológica en los pseudohomófonos. Resalta a su vez un hecho importante, si todos los sujetos hispanohablantes sanos leyeran por mediación fonológica, todos deberían cometer errores como los de MM en la decisión léxica con pseudohomófonos.

Aunque no idéntico a los casos descriptos en sistemas opacos, el caso MM reúne las características de una alexia de superficie. Este caso de alexia de superficie en un paciente hispanohablante puede ser interpretado en el marco de los modelos duales como una disociación entre dos procedimientos de lectura: conservación de los procesos no-léxicos y alteración de los procesos léxicos. El patrón de error en la decisión léxica con pseudohomófonos puede interpretarse como una sobredependencia de 
los mecanismos no léxicos de lectura (mediación fonológica) para realizar tareas cuya realización correcta requiere la utilización de procedimientos léxicos de lectura. La ausencia de errores de regularización en la lectura de palabras irregulares, síntoma característico de la alexia de superficie en lenguas opacas, debe simplemente ser atribuido a que el Español carece de palabras irregulares. Además, MM muestra una escritura realizada por mediación fonológica y que conduce a errores de escritura fonológicamente plausibles, en otras palabras muestra una agrafia de superficie, cuadro que según Coltheart y col. (1983) suele formar parte del complejo sintomático de la alexia de superficie.

Un hallazgo inesperado fue que MM mostró bajo rendimiento en algunas de las tareas que evalúan procesos de la ruta no-léxica. Esto plantea la pregunta ¿cómo logra MM leer por la ruta no-léxica? Nuestra interpretación es que MM conserva un genuino mecanismo de conversión ortografía-fonología a nivel de sílabas y no un mecanismo compensatorio, dependiente de la recuperación léxica como el de LT. De esta manera MM puede destinar recursos para ordenar y pronunciar las sílabas que va obteniendo por conversión, una a una en el caso de las no-palabras, con pronunciación integrada posterior en el caso de las palabras gracias a la activación subsecuente de las representaciones del léxico fonológico de salida.

\section{Alexia profunda}

La alexia profunda es, de todas las alexias adquiridas, la que mayor interés ha concitado. Marshall y Newcombe (1973) publicaron el primer caso. El signo más prominente de la alexia profunda es la producción de paralexias semánticas, error en el que el paciente sustituye la palabra que lee por otra relacionada semánticamente (hierba > campo). Para Coltheart (1980) la producción de paralexias semánticas es el rasgo definitorio de la alexia profunda pero va siempre acompañado por:

1. producción de otros errores paraléxicos (paralexias visuales, morfológicas, sustitución por funcionales),

2. incapacidad para leer no-palabras,

3. mejor lectura de palabras concretas que abstractas (efecto de concretud) y

4. peor lectura de palabras funcionales que de contenido (efecto de clase gramatical).
Se puso en duda la existencia de este cuadro en hispanohablantes con el argumento de la mayor trasparencia del sistema de escritura del Español y el número relativamente bajo de reglas de conversión (unas 50 reglas permiten pronunciar todas las palabras escritas de la lengua mediante procedimientos de conversión letra-sonido). Sin embargo, aunque muy escasos, hay tres pacientes hispanohablantes con alexia profunda publicados, dos de Ruiz, Ansaldo y Lecours (1994) y el paciente JMK estudiado por Ferreres y Miravalles (1995); además de uno comunicado (Dalmás, 1991). Reproducimos a continuación los datos del paciente JMK que son relevantes para este trabajo.

Lectura en voz alta de palabras y nopalabras: JMK mostró una severa alteración en la lectura de palabras y una imposibilidad absoluta para leer no-palabras. Leyó correctamente sólo $21 / 250$ palabras $(8 \%)$ y ninguna no-palabra $(0 \%)$.

La lectura de no-palabras debió ser suspendida luego de seis intentos en los que el paciente no daba ninguna respuesta y debido a su negativa para continuar. La conducta ante palabras y no palabras fue muy distinta y esto se reflejó en la distribución de errores. Frente a las no-palabras decía "estas no", "no puedo", y las omisiones de respuesta representaron el 100\% de los errores. En cambio, intentó leer todas las palabras y, pese a que el porcentaje de correctas fue muy bajo, las omisiones representaron menos de la mitad de las respuestas.

El paciente mostró el efecto de dos variables léxicas en la lectura de palabras, concretud y categoría gramatical, sin embargo estos datos deben tomarse con precaución debido a la falta de control de la frecuencia de los estímulos. Con todo, mostró una marcada diferencia entre nombres concretos ( $26 \%$ de correctos) y abstractos (sólo 4\%). Asimismo los nombres concretos y abstractos fueron mejor leídos que los verbos $(0 \%)$. Un estudio posterior de los datos de JMK realizado por Valle Arroyo (1996) con el Diccionario de Frecuencias de la Lengua Española (Alameda y Cuetos, 1995) verificó que no había un efecto significativo de la frecuencia en el rendimiento.

Patrón de errores en la lectura de palabras, distribución de paralexias: en la lectura de palabras JMK no produjo ninguna no-palabra, 
los errores fueron sustitución por otra palabra (paralexias) u omisiones. Las paralexias más frecuentes fueron las semánticas, seguidas por las morfológicas y las visuales, también presentó paralexias visuales ocultas y sustitución de palabras funcionales.

\section{Discusión del caso JMK}

La imposibilidad de leer no-palabras, la alteración en la lectura de palabras y el predominio de paralexias semánticas en el patrón de error son todos rasgos que corresponden al cuadro de alexia profunda descrito por Marshall y Newcombe (1973). JMK permite documentar que la transparencia del sistema de escritura del español no impide la aparición de pacientes aléxicos que producen gran cantidad de paralexias semánticas y el resto de los síntomas que conforman el complejo sintomático de la alexia profunda.

En el marco de los modelos de doble ruta la alexia profunda se ha interpretado como una alteración que anula completamente la actividad de la ruta no-léxica de lectura, acompañada también por una alteración de la ruta léxica.

La incapacidad absoluta para leer no-palabras sugiere que la vía de mediación fonológica basada en la conversión grafema-fonema estaba completamente alterada y no podía, por lo tanto, ser el medio para acceder al significado de las palabras leídas ni a su pronunciación.

La lectura correcta de algunas palabras sugiere que el paciente conservaba una capacidad residual de lectura léxica. La gran cantidad de paralexias semánticas sugiere que esa capacidad residual de la ruta léxica era suficientemente robusta como para producir errores semánticamente relacionados pero insuficiente para lograr la forma fonológica correcta del blanco, salvo para unos pocos estímulos (el $8 \%$ de palabras leídas correctamente).

Cada paralexia semántica constituye un fenómeno aparentemente paradojal para lectores hispanohablantes, la ortografía de la palabra permite acceder a una cantidad no despreciable de información semántica pero no proporciona ninguna información fonológica del blanco. Es evidente que quien comete un error semántico de lectura, de alguna manera, ha reconocido la palabra y accedido a su significado. Si todo proceso de lectura estuviera fonológicamente mediado, como sostiene Ardila (1991, 1998), este síntoma no se podría producir debido simplemente a que la carencia de información fonológica impediría el acceso a la información semántica. Sólo si se asume que existe un acceso directo a la semántica desde la ortografía, es decir, sólo si se rechaza la mediación fonológica obligatoria, se pueden explicar las paralexias semánticas.

En los modelos de doble ruta, la ruta léxica es la que incluye un acceso directo desde la ortografía a la semántica (Figura 1).

Las paralexias semánticas podrían surgir cuando están alterados algunos de los componentes de la ruta léxica, pero en el marco de una alteración muy severa de la ruta no-léxica; una mínima actividad de la ruta no-léxica proporcionaría la información fonológica suficiente para inhibir la producción de paralexias semánticas (por ejemplo, en el error asno > burro, si la ruta no-léxica proporcionara el fonema /a/, se inhibiría la forma fonológica /burro/ y, probablemente, facilitaría la respuesta correcta, de la misma manera que una clave fonológica mejora la anomia en muchos pacientes o en el fenómeno de la "punta de la lengua" de los sujetos normales).

Otro dato que aporta evidencia a favor del acceso directo desde la ortografía a la semántica es que JMK tuvo un $40 \%$ de acierto en la tarea de emparejamiento palabra escrita/dibujo. Si bien es un rendimiento muy bajo para su nivel de escolaridad - es mucho más alto que el $0 \%$ en la lectura de no-palabras -, muestra que el paciente accedía al significado de palabras escritas de manera suficiente como para señalar el dibujo adecuado y realizar bien el $40 \%$ de los emparejamientos mientras que no podía obtener información fonológica de no-palabras incluso tan pequeñas como una sílaba.

Como señalamos, la ruta léxica no está indemne en JMK. Según el modelo, los déficits podrían hipotéticamente deberse tanto a una alteración del sistema semántico como a una alteración del léxico fonológico de salida. El bajo rendimiento en la tarea de emparejamiento palabra escrita/dibujo ( $40 \%$ de acierto) y la severa anomia de JMK sugieren que tanto el sistema semántico (o su acceso desde la ortografía) como el léxico fonológico (o su acceso desde el sistema semántico) estaban alterados en un grado considerable.

De todos modos, el rendimiento en la tarea de emparejamiento palabra escrita/dibujo y la gran producción de paralexias semánticas en el marco de una incapacidad absoluta para 
obtener fonología desde los estímulos escritos sólo pueden ser explicados postulando un acceso directo (no mediado por la fonología) desde la ortografía al significado. Consecuentemente, la transparencia del español no impide el desarrollo de una ruta (léxica) de acceso directo desde la ortografía al significado.

\section{DISCUSIÓN GENERAL}

Los resultados en lectura de LT, MM y JMK permiten afirmar que existen casos de alexia en hispanohablantes cuyo patrón de alteración coincide con las características de las alexias fonológica, de superficie y profunda descriptos en pacientes con sistemas opacos de escritura. La dificultad inicial para identificar estos patrones entre pacientes hispanohablantes parece más relacionada con la metodología de estudio que con las particularidades de la lengua.

Es posible que los estudios de grupo, que no utilizan las pruebas y estímulos adecuados, y que realizan las inferencias a partir de la promediación de los resultados del grupo, fallen en la identificación de los diferentes patrones.

Sugestivamente, fueron los estudios de caso único, con metodología cognitiva, los que permitieron sacarlos a la luz. Queda pendiente una pregunta de relevancia clínica, ¿cuán frecuentes son los patrones de alexia fonológica, de superficie y profunda entre los pacientes? La metodología de caso único no permite responder esta pregunta. Si la "dificultad para encontrar casos" sirve como sugerencia, hasta que un estudio se dirija a esta cuestión, la alexia fonológica parece el cuadro más frecuente ya que nos resultó más sencillo encontrar aléxicos fonológicos que de superficie y, desde JMK, no hemos observado ningún otro aléxico profundo.

Ardila (1998) sostiene la hipótesis de que el desarrollo de una ruta léxica de lectura en hispanohablantes depende de una exposición no habitual a la lectura. Tanto LT como JMK, los dos pacientes que mostraron mejor lectura léxica que no-léxica, tenían como antecedentes 19 y 12 años de escolaridad formal y una práctica intensa de lectura.Pertenecen sin duda al segmento de la población con niveles más altos de exposición a la lectura, segmento que por cierto no representa a toda la población (aún en países como Argentina que en el pasado mostró altos niveles de alfabetización y de lectura de periódicos y libros), pero es un nivel que de ninguna manera puede ser considerado como excepcional, término que reservaríamos para escritores, periodistas o traductores. Los estudios en lesionados cerebrales con pocos años de escolaridad serían sin duda interesantes pero escasean. La evidencia proveniente de otros campos de investigación también es pertinente para dilucidar la cuestión. ValleArroyo (1996) señaló, a partir del análisis de la eficacia en la lectura de palabras y no-palabras y de los errores en niños hispanohablantes de primero, tercero y sexto grado, que el conocimiento de las reglas de conversión grafema fonema se desarrolla más rápidamente que los procesos de la lectura léxica, que las reglas de correspondencia son casi completamente dominadas en el tercer grado y que en sexto grado la lectura léxica comienza a mostrar sus efectos. Sin duda, una edad y una exposición a la lectura que no coinciden con lo planteado por Ardila (1998).

Una vez identificados en pacientes hispanohablantes los cuadros de alexia fonológica y de superficie, es decir, la doble disociación entre lectura de palabras y no-palabras, los modelos de doble ruta no tienen mayores dificultades para explicar los resultados en español que en otras lenguas. En efecto, la disociación es difícil de explicar salvo que uno asuma que los procesos involucrados en la lectura de palabras son diferentes de los utilizados para leer no-palabras.

Si LT, aléxico fonológico, utilizara exactamente los mismos procesos para leer palabras y no-palabras sería difícil explicar por qué lee bien el $100 \%$ de las palabras y sólo el $60 \%$ de las nopalabras y por qué lee correcta y rápidamente las palabras mientras que tiene que recurrir a una estrategia compleja, laboriosa, lenta y muchas veces errónea para leer no-palabras. En LT, la interpretación más adecuada es que la lesión ha afectado la ruta no-léxica de lectura y a dejado indemne (o poco afectada) la ruta léxica. La alexia fonológica, en español, o en lenguas opacas, no puede ser explicada por el modelo de mediación fonológica obligatoria.

MM, aléxico de superficie, sí parece utilizar el mismo mecanismo para leer palabras y nopalabras: no hay diferencias de tiempo, estrategias, ni eficacia entre la lectura de palabras y no-palabras.

En MM la interpretación más adecuada es que la lesión ha afectado la ruta léxica y dejado indemne (o menos afectada) la ruta no-léxica; 
debido a esto, lee palabras y no-palabras por el mismo procedimiento de conversión, por mediación fonológica.

La teoría de la mediación fonológica obligatoria podría explicar el rendimiento de MM $y$, en este sentido, el caso MM y la alexia de superficie en general, aportan evidencia a favor de la existencia de un procedimiento de lectura por mediación fonológica.

Sin embargo, pese al buen rendimiento en lectura, MM muestra alteraciones muy particulares en la decisión léxica visual, rinde bien con palabras y no-palabras corrientes pero está a nivel del azar en las decisiones léxicas con pseudohomófonos. Nuevamente la mediación fonológica obligatoria podría explicar el resultado, incluso las fallas de MM en pseudohomófonos, precisamente porque la mediación fonológica induce los errores en esta tarea. Pero lo que no puede explicar es por qué un aléxico fonológico como LT no falla con los pseudohomófonos. Más aún, según las predicciones que se derivan de la teoría de la mediación fonológica obligatoria todos los sujetos normales deberían comportarse como MM y fallar en la decisión léxica con pseudohomófonos.

En este sentido la teoría de la mediación fonológica obligatoria explicaría la alexia de superficie (en esto no se diferencia de la teoría de la doble ruta, que también asume que este tipo de alexia el paciente lee por la ruta no-léxica) pero no explicaría la alexia fonológica y no se sostendría como teoría de la lectura normal.

Otro aspecto relacionado es si el acceso al significado está siempre mediado por la fonología tal como sostiene la teoría de la mediación fonológica obligatoria. El caso MM muestra que la mediación fonológica permite acceder al significado ya que el paciente logra un $95 \%$ de acierto en la tarea de emparejamiento palabra escrita/dibujo, aunque previa pronunciación de la palabra de manera abierta o subvocal. Pero pacientes que tienen alteraciones en la mediación fonológica como LT ( $40 \%$ de errores en la lectura de nopalabras) también muestran un buen rendimiento en la tarea de emparejamiento palabra escrita/dibujo, alcanzan el $100 \%$ de aciertos y la realizan de manera más veloz. Más aún, JMK, en el que la mediación fonológica está completamente abolida ya que no logra leer ninguna no-palabra, puede sin embargo acceder al significado de muchas palabras $(40 \%$ de aciertos en la prueba de emparejamiento palabra escrita -dibujo). En consecuencia, tal como lo sostienen los modelos duales, debe postularse una vía de acceso directo desde la ortografía al significado, no mediado por la fonología.

Finalmente, nuestros resultados no rechazan en general la hipótesis de que las particularidades de los sistemas de escritura puedan influir en la arquitectura funcional de los procesos de lectura. Rechazan las suposiciones de que la trasparencia del español impediría el desarrollo de una ruta léxica de lectura y por lo tanto, la aparición de patrones aléxicos como los descriptos para lenguas opacas y aportan evidencia a favor de las explicaciones proporcionadas por los modelos duales. Pero otras características del español, tales como el carácter no ambiguo de su organización silábica así como la riqueza y complejidad de su organización morfológica pueden potencialmente influir en la organización interna de los procedimientos no-léxicos y léxicos respectivamente. Por ejemplo, la estrategia compensatoria utilizada por LT para leer nopalabras, sumamente dependiente de procesos de manipulación silábica, parece más difícil de suponer en un aléxico fonológico inglés que en uno español. Las características de la lengua y la escritura del español deben por lo tanto seguir siendo estudiadas y los pacientes pueden ser una fuente muy rica de evidencias.

\section{REFERENCIAS}

Alameda, J. R. y Cuetos, F. (1995). Diccionario de frecuencias de las unidades lingüísticas del castellano. Oviedo: Servicio de Publicaciones de la Universidad de Oviedo.

Ardila, A.; Rosselli, M.; y Pinzon, O. (1989). Alexia and agraphia in Spanish speakers. En A. Ardila y F. Ostrosky-Solis (eds.), Brain Organization of Language and Cognitive Processes. Nueva York: Plenum Press.

Ardila, A. (1991). Errors resembling semantic paralexias in Spanish-speaking aphasics. Brain and Language, 41, 437-445.

Ardila, A. (1998). Semantic paralexias in Spanish language. Aphasiology, 12, 885-90.

Beauvois, M. F. y Dérouesné, J. (1989). Phonological alexia: Three dissociations. Journal of Neurology, Neurosurgery and Psychiatry, 42, 1115-1124.

Bernd, R.; Haendiges, A.; Mitchum, C.; Wayland, S. (1996). An investigation of nonlexical reading impairments. Cognitive Neuropsychology, 13, 763-801. 
Coltheart, M. (1980). Deep Dyslexia: a review of the syndrome. En M. Coltheart, K. Patterson y J. Marshall (Eds.), Deep Dyslexia. Londres: Routledge.

Coltheart, M. (1996). Phonological dyslexia: past y future issues. Cognitive Neuropsychology, 13, 749762.

Coltheart, M.; Patterson, K. y Marshall, J. (1980). Deep Dyslexia. Londres: Routledge y Keagan Paul.

Coltheart, M.; Curtis, B.; Atkins, P. y Haller, M. (1993). Models of reading aloud: Dual route and parallel distributed processing approach. Psychological Review, 100, 589-608.

Cuetos, F.;Valle-Arroyo, F.; y Suárez, M. (1996). A case of phonological Dyslexia in Spanish. Cognitive Neuropsychology, 13, 1-24.

Dalmás, F. (1991). Alexia profunda. Los caminos de la lectura. Trabajo presentado en el II Congreso Latinoamericano de Neuropsicología. San Pablo, Noviembre de 1991.

Dansilio, S \& Dalmás, L. (1997). Alexia fonológica (casi profunda) en castellano. Neuropsychologia Latina, 2, 85

Dubrovsky, S., Kolesnikof O., Miravalles G., y Ferreres A. (1990). Protocolo de lectura de palabras aisladas. No publicado.

Ellis, A. W. (1992). Normality and pathology in cognitive functions. Londres: Academic Press.

Ferreres, A. y Miravalles, G. (1995). The production of semantic paralexias in Spanish speaking aphasic. Brain and Language, 49, 153-172.

Ferreres, A.; Grus, J.; Jacubovich, S.; Jaichenco, V.; Kevorkian, A.; Piaggio, V.; Politis, D.; Recio, F. (1999). Batería para el Análisis de los Déficits Afásicos. Buenos Aires: JVE ediciones. Versión en español de la Battería per l'analisi dei deficit afasici de G. Miceli , A. Laudanna, C. Burani y C. Capasso. Berdata: Milan, 1991.

Ferreres,A., Martinez Cuitiño, M., Jacubovich, S., Olmedo, A. y López, C. (2003). Las alexias y los modelos de doble ruta de lectura en hispanoparlantes. Revista Argentina de Neuropsicología 1, $37-52$.

Ferreres, A., López, C. \& China, N. (2003). Phonological alexia with vowel-consonant dissociation in nonword reading. Brain \& Language 84, 399-413.
Ferreres, A., Martinez Cuitiño, M., Olmedo, A. (2005). Acquired surface alexia in Spanish: A case report. Behavoiural Neurology,16, 71-84. ISSN: 0953-4180. IOS Press.

Ferreres, A. (2007). Evaluación de las alteraciones de la lectura. En D. Burin, M. Drake y P. Harris (compiladoras) Evaluación Neuropsicológica en Adultos. Buenos Aires: Paidos. ISBN 978-950-12-6084-7.

Ferreres, A., China, N. y Abusambra, V. (2008). Las afasias. En E. Labos, A. Slachevsky, P. Fuentes y F. Manes (Eds) Tratado de Neuropsicología clínica. Buenos Aires: Akadia Editorial.

Gough, P. B. (1972). One second of reading. En J.F Kavanagh y G. Mattingly (Eds.) Language by ear and by eye. Cambridge, Mass.: MIT.

Miceli, G.; Capasso, R.; Caramazza, A. (1994). The interaction of lexical and sublexical processes in reading, writing and repetition. Neuropsychologia, 32, 317-333.

Patterson K., Marshall J., Coltheart M. (1985). Surface Dyslexia. Neuropsychological and cognitive studies of phonological reading. Londres: Lawrence Erlbaum Associates.

Patterson, K.E. y Morton, J. (1985). From orthography to phonology: an attempt at an old interpretation. En K.E. Patterson, J.C. Marshall y M. Coltheart (Eds.) Surface Dyslexia. Neuropsychological and cognitive studies of phonological reading. Londres: Lawrence Erlbaum Associates.

Ruiz, A.; Ansaldo, A. y Lecours, A. R. (1994). Two cases of deep dyslexia in unilingual hispanophone aphasics. Brain and language, 46, $244-256$.

Sartori, G.; Barry, C. y Remo, J. (1984). Phonological Dyslexia: a review. En R.N. Malatesha y H.A. Whitaker (Eds.) Dyslexia: a Global Issue. La Haya: Martinus Nijhoff.

Valle-Arroyo, F. (1996). Dual-route models in Spanish: developmental and neuropsychological data. En M. Carreiras, J. García Albea, N. SebastiánGallés (Eds.). Language processes in Spanish. Nueva Jersey: LEA.

Van Orden, G.C.; Johnston, J.C. y Hale, B. I.. (1988). Word idenitifcation in reading proceeds form spelling to sund to meaning. Journal of Experimental Psychology: Learning, Memory and Cognition, 14, 371-385. 